\title{
Surface-Functionalized Silk Fibroin Films as a Platform To Guide Neuron-like Differentiation of Human Mesenchymal Stem \\ Cells
}

Shivaprasad Manchineella, ${ }^{\dagger, t}$ Greeshma Thrivikraman, ${ }^{\xi, t}$ Bikramjit Basu $u^{\xi *}$ and T.

Govindaraju (†* $^{* *}$

${ }^{\dagger}$ Bioorganic Chemistry Laboratory, New Chemistry Unit, Jawaharlal Nehru Centre for Advanced Scientific Research, Jakkur, Bengaluru 560064, Karnataka, India.

*E-mail: tgraju@jncasr.ac.in (T. Govindaraju)

${ }^{\S}$ Laboratory for Biomaterials, Materials Research Centre, Indian Institute of Science, Bengaluru 560012, Karnataka, India.

*Email: bikram@mrc.iisc.ernet.in (B. Basu) 

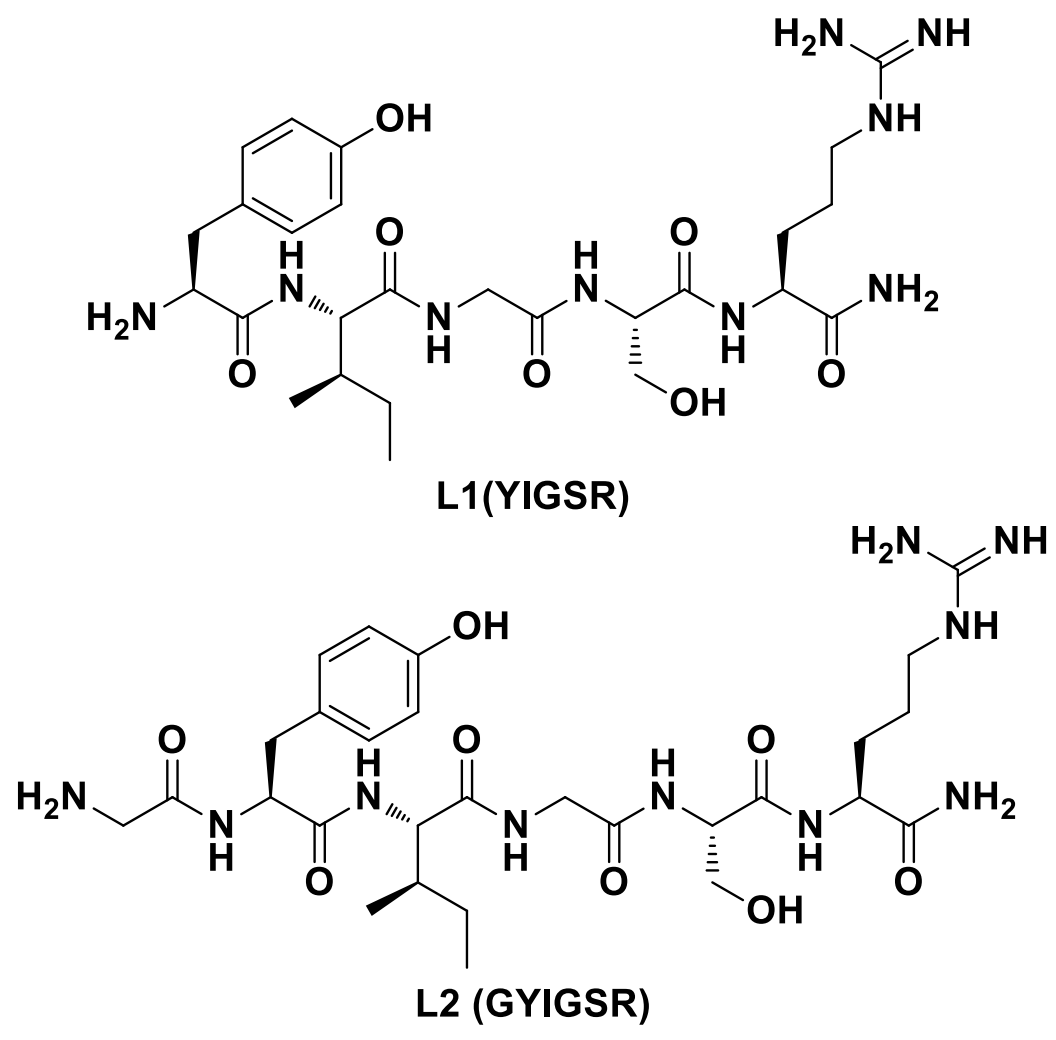

Scheme S1. Chemical structures of integrin binding laminin derived peptide motifs. L1: YIGSR; L2: GYIGSR. 


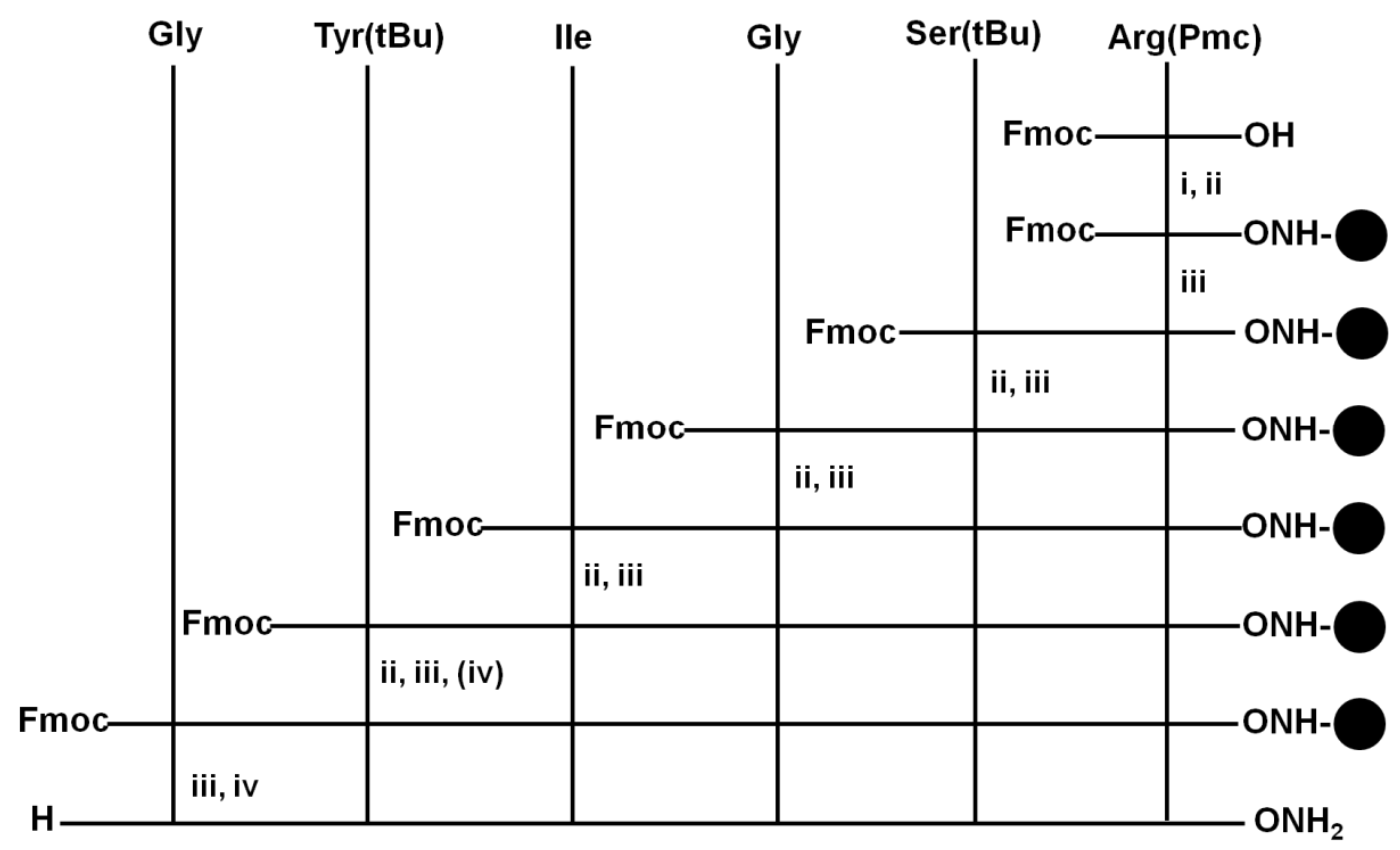

Figure S1. Schematic showing solid phase peptide synthesis (SPPS) of peptides L1 (YIGSR) and L2 (GYIGSR) rink amid resin. (i) Fmoc-deprotected rink amide resin; (ii) HBTU in DMF, DIPEA in NMP; (iii) 40\% piperidine in DMF; (iv) TFA: $\mathrm{H}_{2} \mathrm{O}$ :TIPS (90:05:05) and the synthesis was carried out under ambient conditions. HBTU: O-(benzotriazol-1-yl)- $N, N, N$, $N$ ' tetramethyluronium hexafluorophosphate; DIPEA: $N, N$-diisopropylethylamine ; DMF: $N, N$-dimethylformamide; NMP: $N$-Methyl-2-pyrrolidone; TFA: Trifluoroacetic acid; TIPS: Triisopropylsilane. 


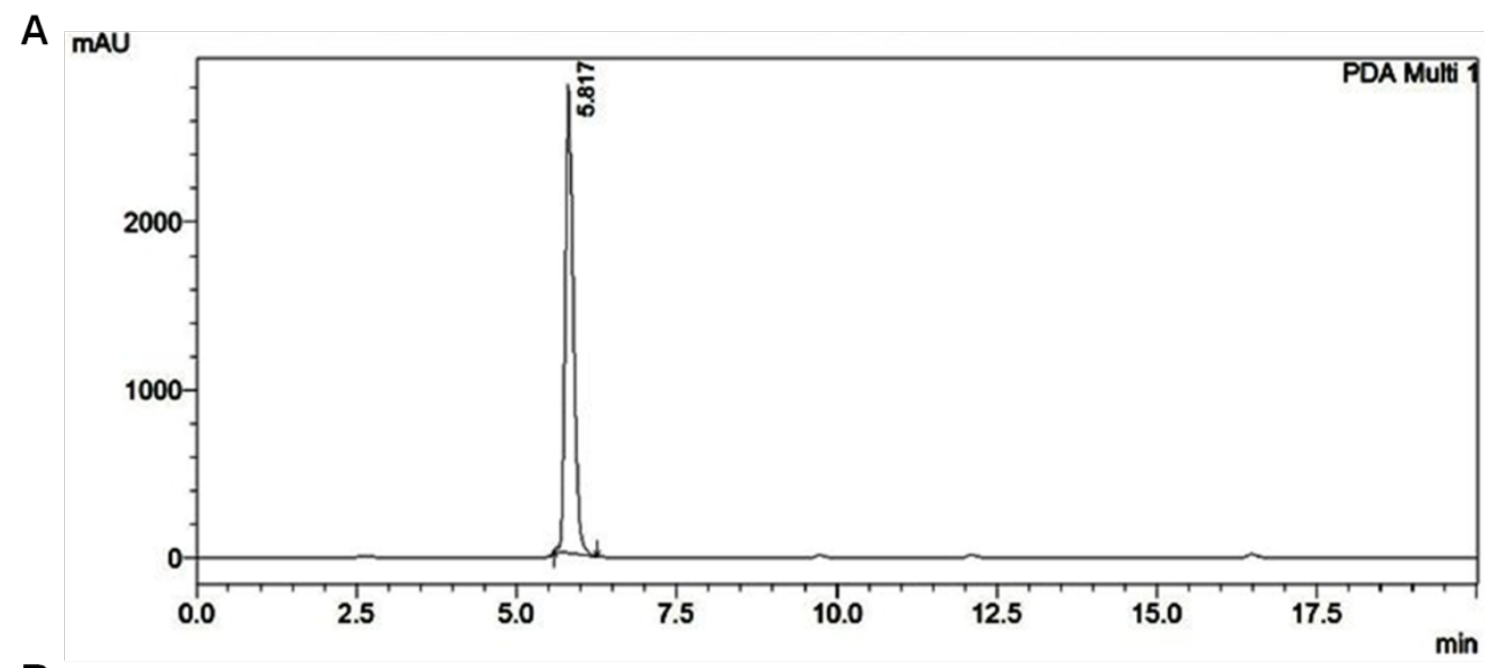

B

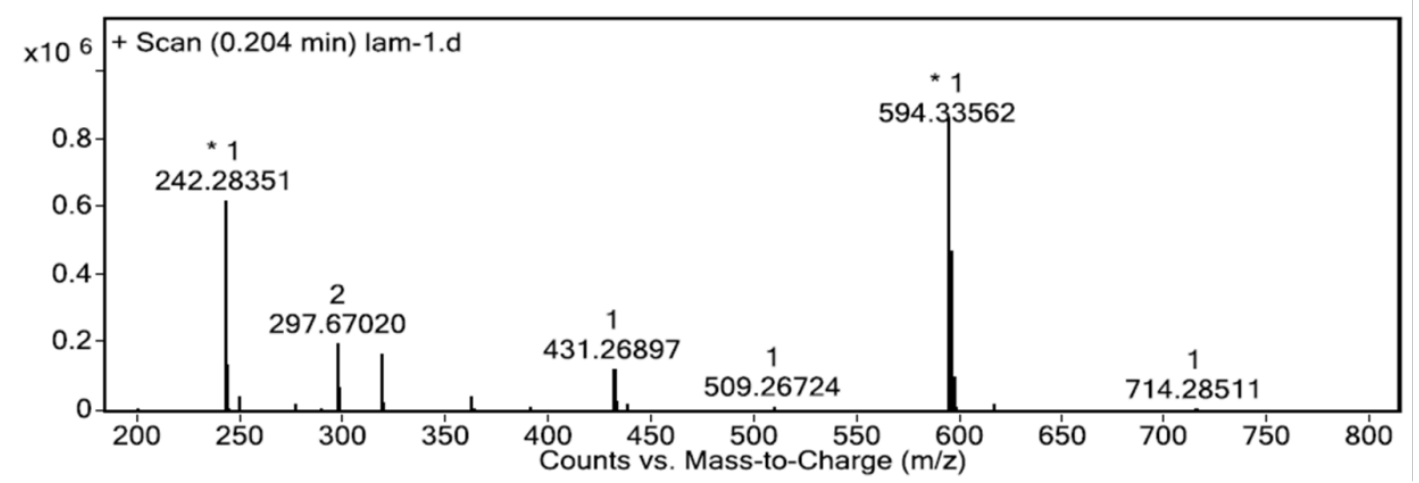

Figure S2. Characterization of L1 (YIGSR). (A) HPLC chromatogram showing the purity of L1. The chromatogram absorption was monitored at $215 \mathrm{~nm}$ using PDA detector. (B) HRMS spectra of L1. HRMS (ESI): m/z calcd for $\mathrm{C}_{26} \mathrm{H}_{44} \mathrm{~N}_{9} \mathrm{O}_{7}: 594.3364[\mathrm{M}+\mathrm{H}]^{+}$; found: 594. 3356. 


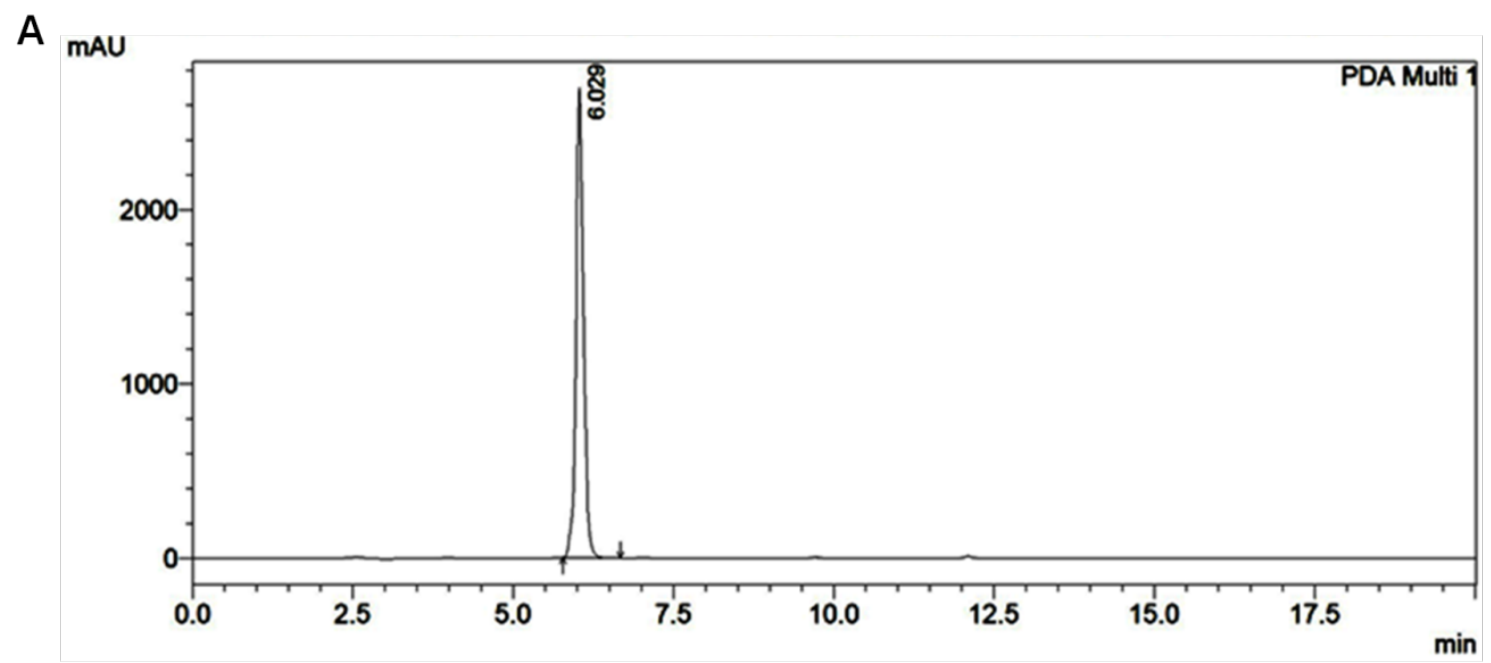

B

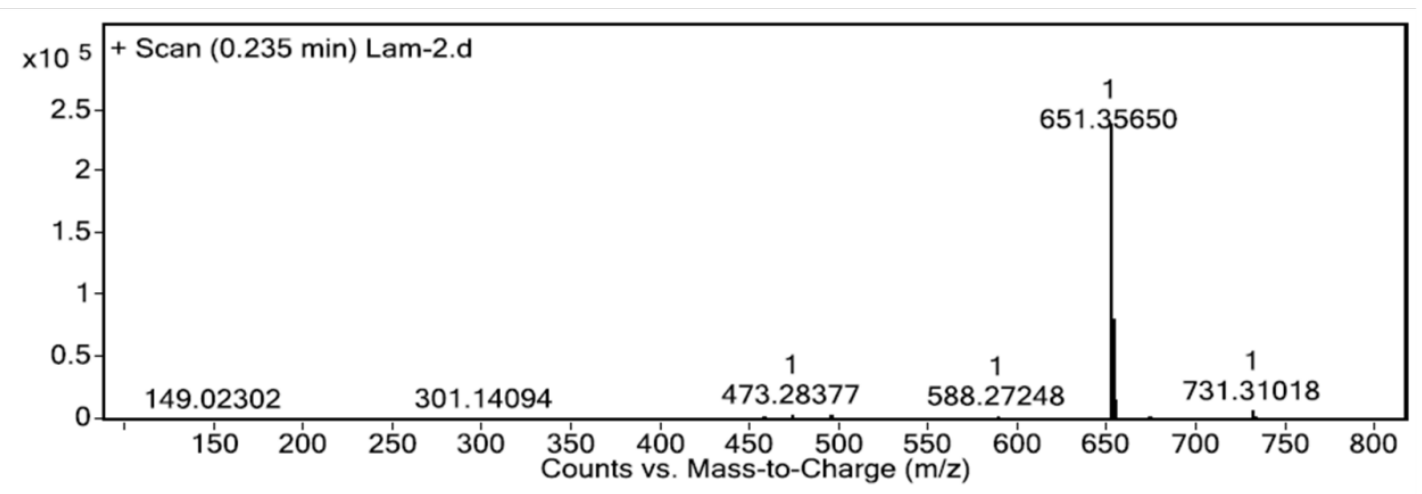

Figure S3. Characterization of L2 (GYIGSR). (A) HPLC chromatogram showing the purity of L2. The chromatogram absorption was monitored at $215 \mathrm{~nm}$ using PDA detector. (B) HRMS spectra of L2. HRMS (ESI): m/z calcd for $\mathrm{C}_{26} \mathrm{H}_{47} \mathrm{~N}_{10} \mathrm{O}_{8}: 651.3578[\mathrm{M}+\mathrm{H}]^{+}$; found: 651.3565 . 


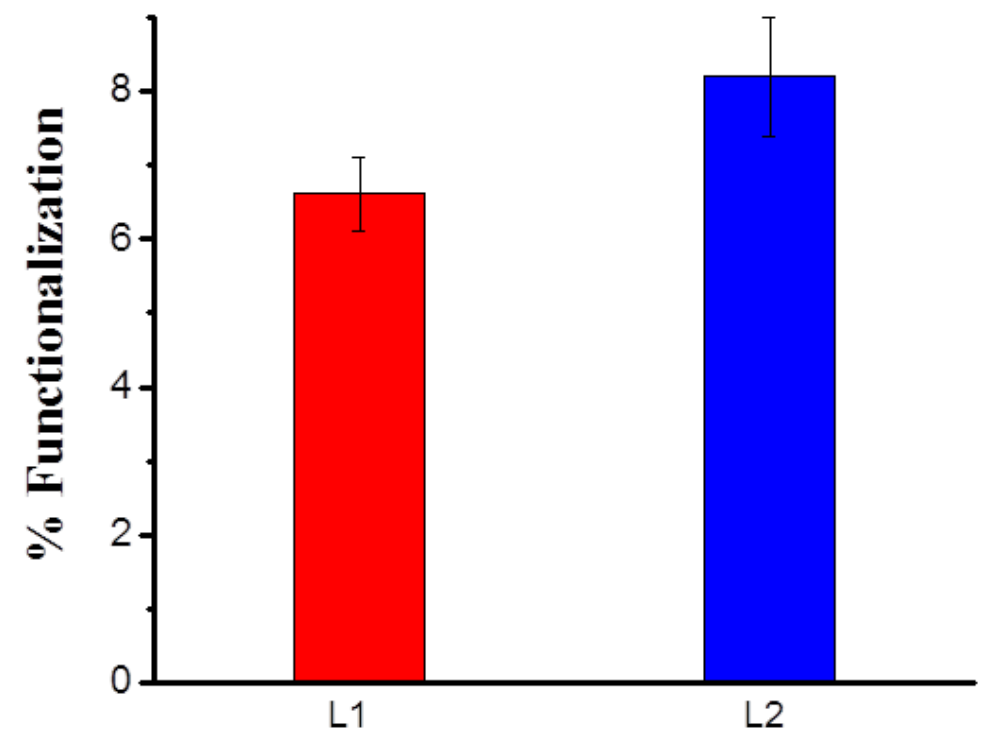

Figure S4. UV-vis absorption analysis of \% covalent functionalization of peptides L1 and L2 on SFFs. Absorption intensity of supernatant is monitored at $275 \mathrm{~nm}$ and \% functionalization is calculated using standard peptide solution. 
Table S1. Hydrophilicity of pristine and surface functionalized SFFs. Contact angle values for water and $\alpha \mathrm{MEM}$ droplets on the surface of SFFs measured under ambient conditions.

\begin{tabular}{lcc}
\hline Sample & $\begin{array}{c}\mathbf{H}_{2} \mathbf{O} \\
\text { Contact angle }\end{array}$ & $\begin{array}{c}\boldsymbol{\alpha M E M} \\
\text { Contact angle }\end{array}$ \\
\hline UM-SFF & $64.6 \pm 0.1$ & $55.9 \pm 0.9$ \\
PL1-SFF & $64.5 \pm 0.8$ & $53.1 \pm 0.9$ \\
CL1-SFF & $61.2 \pm 0.6$ & $55.9 \pm 0.9$ \\
PL2-SFF & $61.9 \pm 0.6$ & $50.7 \pm 0.2$ \\
CL2-SFF & $60.6 \pm 0.6$ & $54.9 \pm 0.7$ \\
Lam-SFF & $65.4 \pm 0.2$ & $59.9 \pm 0.8$ \\
\hline
\end{tabular}

Table S2. List of primers used for the semi-quantitative RT-PCR neuronal specific gene expression analysis. NES: nestin; TUBB3: class-III beta tubulin; NEFL: neurofilament light polypeptide; MAP2: microtubule-associated protein 2.

\begin{tabular}{|c|c|c|c|}
\hline \multirow[t]{2}{*}{ Genes } & \multicolumn{2}{|c|}{ Primer Sequence } & \multirow{2}{*}{$\begin{array}{l}\text { Amplicon } \\
\text { Size (bp) }\end{array}$} \\
\hline & Forward & Reverse & \\
\hline GAPDH & AGGTCGGTGTGAACGGATTTG & TGTAGACCATGTAGTTGAGGTCA & 123 \\
\hline NES & AACAGCGACGGAGGTCTCTA & TTCTCTTGTCCCGCAGACTT & 220 \\
\hline TUBB3 & ATGTGGTGCGGAAGGAGTGTGAAA & TGTTCATGATGCGGTCGGGATACT & 144 \\
\hline NEFL & ACCTCCTCAACGTGAAGATGGCTT & ACTCTTCCTTGGCAGCTTCTTCCT & 384 \\
\hline MAP2 & TAACCAACCACTGCCAGACCTGAA & GCCACATTTGGATGTCACATGGCT & 232 \\
\hline
\end{tabular}

